\title{
THE CONTROL OF THE CONSUMER RISK IN THE AMES ASSAY
}

\author{
MARKUS NEUHÄUSER \\ Solvay Pharmaceuticals, Hannover, Germany \\ LUDWIG A. HOTHORN \\ Universität Hannover, LG Bioinformatik, Hannover, Germany
}

\begin{abstract}
Preclinical tests in genetic toxicology represent safety studies. Therefore, the primary concern in the evaluation of Ames test data is the control of the consumer risk, that is, the risk of erroneously concluding safety. Hence, an equivalence test procedure is adequate. This approach is presented beside the two-fold rule and classical tests for differences with respect to an order restricted alternative.
\end{abstract}

Key Words: Ames assay; Consumer risk; Difference approach; Equivalence test procedures for ordered many-to-one designs

\section{INTRODUCTION}

THE AMES (1) ASSAY IS a short-term test in genetic toxicology (2) most often used worldwide. This assay is based on the detection of mutated histidine-dependent cell strains of Salmonella typhimurium. The mutated bacteria can mutate back to the wild-type, particularly if they are exposed to mutagenic compounds. The endpoint for evaluation is the number of revertant colonies on replicated plates. This endpoint is a count.

There are some difficulties in the statistical evaluation of the Ames assay:

- As the distribution of the discrete endpoint is not clear, different distributions are used: the Poisson distribution and two special cases of the generalized Poisson distribution according to Consul and Jain (3), the negative binomial distribution is an extension to the Poisson distribution as well (4). Furthermore, the normal distribution is used after a suitable transformation,

- Small and unbalanced sample sizes are used according to guidelines, for example, three per dose group and five for the negative control group are common sample sizes. With the small sample sizes, the variance may be reduced by ties. As a consequence, this can lead to a false positive result,

- The variances are frequently nonhomogeneous because of small sample sizes and ties. Moreover, the variance can increase with increasing effects, and

Presented at the DIA Workshop "Statistical Methodology in Non-Clinical and Toxicological Studies," March 25-27, 1996, Bruges, Belgium.

Reprint address: Markus Neuhäuser, Solvay Pharmaceuticals, Hans-Böckler-Allee 20, D-30173 Hannover, Germany. 
- There is the possibility of a downturn at high doses. This so-called muta-tox problem should be taken into account. There are different statistical approaches: One can ignore the muta-tox problem; this strategy is frequent in praxi. Another pragmatic method is to reject high doses before testing (5). A transformation can moderate the muta-tox problem. Moore and Felton (6) propose a weighted regression. Another method is stepwise testing. There are step-up and step-down procedures $(7,8)$. Chen (9) proposes trend tests for umbrella alternatives. One can test a priori ordered hypotheses based on linear contrasts according to Rom et al. (10). In the authors' example they use a modification of the Shaffer-II procedure (11). The last two approaches are not special tests for umbrella alternatives, but they are robust against umbrella alternatives because of the partial ordered alternative.

A pragmatic approach to analyze Ames assay data is the two-fold rule (12). One decides in favor of mutagenicity if at least two nonzero doses produce a result more than two-fold the spontaneous background. Sakomoto (13) wrote that: "In Japan, the data obtained in the Ames test are empirically evaluated using the two-fold rule . . . because there is no broadly accepted consensus of statistical evaluation for mutagenicity testing." The two-fold rule has two main disadvantages:

1. The spontaneous background depends on the strain. Consequently, for strains with a high spontaneous background, for example, TA 100, the two-fold rule is particularly conservative, the type one error rate is nearly zero (13). As a result, the type two error rate, the consumer risk, is too high, and

2. The variability depends on the strain, too. Hence, the two-fold rule does not take statistical considerations into account.

Nonparametric trend tests or two-sample tests in a closed test procedure are recommended. The authors use the Jonckheere (14) trend test and the rank transform test for heterogeneous variances (15). The Jonckheere trend test is available in SAS; an asymptotic approximation can be performed by the SAS procedure CORR (16). Berry (17) presented an SAS/TML program to obtain (simulation-based) exact significance levels.

These tests were illustrated with real Ames test data (Table 1). In this example one negative control group and five dose groups were tested in a many-to-one design.

\section{THE TRADITIONAL DIFFERENCE APPROACH}

First the data of Table 1 were analyzed with the traditional difference approach. A modification of the Shaffer-II procedure (11), a closure principle for partial ordered hypotheses, is used with the Jonckheere trend test. The null hypothesis is the equality of the means:

TABLE 1

Revertant Counts of TA 1535 Strains without Metabolic Activation (18)

\begin{tabular}{cccccc}
\hline Dose (in $\mu \mathrm{g})$ & \multicolumn{5}{c}{ Number of Revertants } \\
\hline 0 & 16 & 17 & 17 & 20 & 18 \\
5 & 18 & 18 & 19 & & \\
15 & 16 & 20 & 20 & & \\
50 & 20 & 24 & 28 & & \\
150 & 26 & 28 & 20 & & \\
500 & 16 & 20 & 16 & & \\
\hline
\end{tabular}




$$
\mathrm{H}_{0}^{i}: \mu_{\mathrm{C}^{-}}=\mu_{1}=\ldots=\mu_{i}, i \in\{1,2,3,4,5\}
$$

where $\mu_{\mathrm{C}-}$ denotes the location parameter for the negative control group and $\mu_{j}, j \geq 1$, for the dose groups. The null hypothesis is tested versus the following one-sided ordered alternative:

$$
H_{1}^{i}: \mu_{\mathrm{C}-} \leq \mu_{1} \leq \ldots \leq \mu_{i} \text { with at least } \mu_{\mathrm{C}-}<\mu_{i}, i \in\{1,2,3,4,5\}
$$

In the first step the Jonckherre $P$-value is computed for all groups, and without the highest dose group, and without the two highest doses, and so forth. Altogether, there are five $P$-values in the first step (Table 2). The smallest $P$-value is smaller than $\alpha / 5=0.01$. Thus, the global null hypothesis is rejected, and one can go to the second step.

In the second step the dose with the smallest $P$-value and all higher doses are eliminated. Thus, there are only three $P$-values (Table 2 ). The smallest is smaller than $\alpha / 3=0.017$. In the third step the dose 50 is eliminated and there are two $P$-values (Table 2). The smallest is greater than $\alpha / 2=0.025$. Thus, the procedure must be stopped, and a global dose-response effect has been proven. The lowest significant dose is $50 \mu \mathrm{g}$.

In a pairwise contrast one compares the negative control group with the highest dose group:

$$
\mathrm{H}_{0}^{i}: \mu_{\mathrm{C}-} \geq \mu_{i} \quad \text { versus } \quad \mathrm{H}_{1}^{i}: \mu_{\mathrm{C}-}<\mu_{i}, i \in\{1,2,3,4,5\}
$$

With these hypotheses and the rank transform test (15) the same lowest significant dose results as with the Jonckheere test.

\section{EQUIVALENCE TESTS}

Concerning drugs, the primary concern is the control of the consumer risk (erroneously concluding safety). Therefore, one does not want to prove mutagenicity, one wants to exclude mutagenicity. Consequently, the aim is not to show a significant difference but equivalence,

TABLE 2

Evaluation of the Ames Assay Data

\begin{tabular}{llc}
\hline Step & Included Doses & $\begin{array}{c}\text { Jonckheere } P \text {-Value } \\
\text { (exact, one-sided) }\end{array}$ \\
\hline 1st step & {$[0,5,15,50,150,500]$} & $P=0.0509$ \\
& {$[0,5,15,50,150]$} & $P=0.0003$ \\
& {$[0,5,15,50]$} & $P=0.0045$ \\
& {$[0,5,15]$} & $P=0.1335$ \\
& {$[0,5]$} & $P=0.1964$ \\
2nd step & $P_{\min }=0.0003<\alpha / 5=0.01$ & \\
& {$[0,5,15,50]$} & $P=0.0045$ \\
& {$[0,5,15]$} & $P=0.1335$ \\
3rd step & {$[0,5]$} & $P=0.1964$ \\
& $P_{\min }=0.0045<\alpha / 3=0.017$ & \\
& {$[0,5,15]$} & $P=0.1335$ \\
& $P_{\min }=0.1335>\alpha / 2=0.025$ & $P=0.1964$ \\
\hline
\end{tabular}


the aim is the so-called proof of safety (19). A one-sided equivalence procedure for ordered hypotheses based on pairwise contrasts (18) is used and the following hypotheses are tested:

$$
\mathbf{H}_{0}^{i}: \mu_{\mathrm{C}-}+\varepsilon \leq \mu_{i} \quad \text { versus } \quad \mathbf{H}_{1}^{i}: \mu_{\mathrm{C}_{-}}+\varepsilon>\mu_{i}, i \in\{1,2,3,4,5\}
$$

In the first step of a closed testing procedure the negative control $\mathrm{C}$ - is compared with the lowest dose at level $\alpha$. If equivalence can be proven the negative control is compared in the second step with the second dose at level $\alpha$, and so forth. All tests can be performed with level $\alpha$ without adjustment.

With an a priori defined shift parameter $\varepsilon=5$ and the rank transform test the following $P$-values are computed:

- $\mathrm{C}-$ versus dose 1: $P<0.0001$,

- $\mathrm{C}-$ versus dose 2: $P<0.0001$,

- $\mathrm{C}$ - versus dose 3: $P=0.4165$,

- C- versus dose 4: $P=0.3727$, and

- $\mathrm{C}$ - versus dose 5: $P<0.0001$.

In both the first and second steps the $P$-value is smaller than $\alpha=0.05$. In the third step the $P$-value is greater than $\alpha=0.05$, and the procedure stops. Mutagenicity can be excluded for the first and second doses. The no-observed-effect-dose is $15 \mu \mathrm{g}$. The agreement with the difference approach is not compelling, of course, it depends on the shift parameter $\varepsilon$.

\section{CONCLUSIONS}

Tests in genetic toxicology represent safety studies. Therefore, the consumer risk has to be controlled in an Ames assay. One has to carry out equivalence tests. This can be lead to a controversy about the one-sided equivalence region $\varepsilon$ which has to be chosen. An equivalence region can be chosen based on the two-fold rule or information about historical controls. In addition to the traditional difference approach, a proof-of-safety test was presented for the evaluation of Ames assay data.

\section{REFERENCES}

1. Ames BN, McCann J, Yamasaki E. Methods for detecting carcinogens and mutagens with the Salmonella/ Mammalian-microsome mutagenicity test. Mutation Res. 1975;31:347-364.

2. Edler L. Statistical methods for short-term tests in genetic toxicology: The first fifteen years. Mutation Res. 1992;277:11-33.

3. Consul P, Jain G. A generalization of the Poisson distribution. Technometrics. 1973;15:791-799.

4. Broekhoven LH, Nestmann ER. Statistical analysis of the Salmonella mutagenicity assay. In: Krewski D, Franklin C, eds. Statistics in toxicology. London: Gordon and Breach; 1991:205-264.

5. Chu KC, Patel KM, Lin AH, Tarone RE, Lin MS, Dunkel VC. Evaluating statistical analyses and reproducibility of microbial mutagenicity assays. Mutation Res. 1981;85:119-132.

6. Moore D, Felton JS. A microcomputer program for analyzing Ames test data. Mutation Res. 1986;119:95-102.

7. Simpson DG, Margolin BH. Nonparametric testing for dose-response curves subject to downturns: Asymptotic power considerations. Ann Stat. 1990;18:373-390.

8. Schmoor C, Schumacher M. Adaptive statistical procedures for the analysis of nonmonotone dose-response relationships. Biometrie Informatik Medizin Biologie. 1992;23:113-126.

9. Chen YI. Nonparametric comparisons of umbrella pattern treatment effects with a control in a one-way layout. Comm Stat-Simula. 1995;B22:749-764.

10. Rom DM, Costello RJ, Connell LT. On closed test procedures for dose-response analysis. Stat Med. 1994;13: 1583-1596.

11. Tamhane AC, Hochberg Y, Dunnett CW. Multiple test procedures for dose finding. Biometrics. 1996;52:21-37. 
12. McCann J, Hom L, Kaldor J. An evaluation of Salmonella (Ames) test data in the published literature: Application of statistical procedures and analysis of mutagenic potency. Mutation Res. 1984;134:1-47.

13. Sakomoto Y, Hamada C, Wada T. Statistical characterization of negative control data in the Ames test. Mutation Res. 1992;272:280.

14. Jonckheere AR. A distribution-free $k$-sample test against ordered alternatives. Biometrika. 1954;41:133-145.

15. Akritas MG, Arnold SS, Brunner E. Non-parametric hypotheses and rank statistics for unbalanced factorial designs. J Am Stat Assoc. 1997.

16. Morris RW, Dietz EJ. How to perform Jonckheere's test using the CORR procedure. Proceedings of the 14th annual SAS users group international conference: 1989;1337-1339.

17. Berry JJ. A simulation-based approach to some nonparametric statistics problems. Observations. 1995;5: 19-26.

18. Neuhăuser M, Hothorn LA. Auswertung der Dosis-Wirkungs-Abhängigkeit des Ames Mutagenitätsassay bei direkter Kontrolle des Konsumentenrisikos. In: Trampisch HJ, Lange S, eds. Medizinische Forschung- ̈̈rztliches Handeln. Munich: MMV Medizin Verlag; 1995:113-116.

19. Hauschke D. Statistical proof of safety in preclinical testing. Drug Inf J. 1997;31. 